\title{
Effect of irradiated chitosan treatment on storage life of fruits of two commercially grown papaya (Carica papaya L.) varieties ${ }^{* *}$
}

\author{
I.G.N. Hewajulige ${ }^{1 *}$, Y. Sultanbawa ${ }^{1}$, R.S. Wilson Wijeratnam ${ }^{1}$ and R.L.C. Wijesundara ${ }^{2}$ \\ IFood Technology Section, Industrial Technology Institute (ITI), 363, Bauddhaloka Mawatha, Colombo 07. \\ 2 Department of Plant Sciences, Faculty of Science, University of Colombo, Colombo 03.
}

Revised: 03 August 2008 ; Accepted: 18 November 2008

\begin{abstract}
Potential use of gamma irradiated chitosan for control of the anthracnose disease causing organism, Colletotrichum gloeosporioides, and extension of storage life of papaya varieties Rathna and Red Lady were investigated. Chitin extracted from shrimp waste was converted into chitosan and used as an antifungal treatment against fungal strains isolated from both papaya varieties. Chitosan in powder form and as $1 \%$ solution were irradiated with different doses $-5,10$, $25,50,75,100$ and $150 \mathrm{kGy}$ of ${ }^{60} \mathrm{Co}$ gamma rays-in order to enhance antifungal activity. The effective irradiation dose for the inhibition of radial mycelial growth of respective fungal strains was selected via a series of in vitro experiments using potato dextrose agar (PDA). A complete inhibition of growth in both fungal strains was observed on PDA plates incorporated with $1 \%$ chitosan solution at all irradiated doses compared to the control (distilled water). The plates incorporated with nonirradiated chitosan $(0 \mathrm{KGy})$ and inoculated with the fungus isolated from Red Lady papaya showed slight mycelial growth while Ratna isolate showed complete inhibition of growth with this treatment. However, irradiated chitosan powder was found to be less effective in inhibiting in vitro growth of both fungal strains, $1 \%$ chitosan solution (in 10\% acetic acid) irradiated at the lowest dose ( $5 \mathrm{kGy}$ ) was selected for in vivo experiments on storage life extension of papaya fruits. Eighty percent marketable quality fruits were observed in Rathna papaya while $70 \%$ marketable quality fruits were observed in Red Lady subjected to irradiated chitosan treatment and stored at $13.5^{\circ} \mathrm{C}, 95 \% \mathrm{RH}$ for 14 days followed by 2 days at ambient temperatre $\left(28^{\circ} \mathrm{C} \pm 2\right)$.
\end{abstract}

Keywords: Antifungal activity, chitosan, Colletotrichum gloeosporioides, Papaya, irradiation, Rathna, Red Lady Papaya.

\section{INTRODUCTION}

Chitosan is used as a natural coating for fruit and is derived from crab or prawn shell after deacetylation of chitin. Next to cellulose it is the second most abundant, naturally available, easily degradable biopolymer. Chitosan is known to form a semi permeable film, which modifies the internal atmosphere of the fruit and decreases transpiration losses. It is known to lower the rate of respiration and delay the ripening process. Chitosan shows antifungal activity by inducing the defense barrier in host tissue ${ }^{1}$. Chitosan thus helps to retain the quality of fruits during storage and is non toxic and biologically $\mathrm{safe}^{2}$. Previous studies ${ }^{3,4}$ revealed that chitosan extracted from locally available head and shell waste of farm shrimps - at a concentration of $1 \%$ could be used to extend the storage life (with $70 \%$ marketability) of papaya var. Rathna up to 14 days at $13.5^{\circ} \mathrm{C}$ and $95 \%$ relative humidity. However, $1 \%$ chitosan dip treatment was not effective in controlling the anthracnose disease in papaya var. Red Lady that was introduced recently to Sri Lanka (authors' unpublished data).

Biological activity of chitosan depends significantly on its physico-chemical properties such as molecular weight and the molecular fraction of glucosamine units in the chitosan polymer chain. Hence, the application of this polysaccharide is limited in some fields due to its high molecular weight resulting in low solubility in aqueous diluted acids and high viscosity of the solution even at low concentrations ${ }^{5}$. Ionization radiation is known as an effective process in the modification of polymers ${ }^{6}$. The

\footnotetext{
* Corresponding author (ilmi@iti.lk)

** Part of this work was presented at the Annual Sessions of the Institute of Biology in 2007.
} 
two main reactions, which determine the final properties of a polymer, include (a) main chain scission leading to the reduction of molecular weight (b) cross linking the opposite process, resulting in formation of macroscopic insoluble material.

Chitosan belongs to the class of natural polymers that undergoes depolymerization when exposed to ionization radiation $^{7,8}$. Previous studies ${ }^{9}$ have investigated effects of $\gamma$-irradiation on chitosan fibres and films, and observed that the viscosity, average molecular weight of the polymer decreased with increasing irradiation dose and radiation induced scission of the chitosan chain. Low molecular weight chitosan has wide application as a fungicide. In Thailand irradiated chitosan fruit coating is used to eliminate some fungi on the skin as well as to preserve the texture by reducing respiration and water evaporation in Salak, Salacca wallichina ${ }^{10}$. Mango coated with irradiated chitosan (in India) showed extended shelf life from 7-15 days (Personal communication: A.G. Chemielewski International Atomic Energy Authority, Vienna, 2005). However, studies on postharvest application of irradiated chitosan are limited. The objectives of the present investigation are to determine the antifungal activity of irradiated chitosan on the anthracnose causing fungal strains isolated from papaya and to test the efficacy of irradiated chitosan as a fruit coating to extend the storage life of papaya.

\section{METHODS AND MATERIALS}

Extraction of chitosan: Chitin was extracted from prawn shell and head waste at the Fish Technology Laboratory, Industrial Technology Institute (ITI). Extraction process (ITI patent pending for the method of extraction) included deproteinization with $5 \% \mathrm{NaOH}$ and demineralization with $2 \% \mathrm{HCl}$. Extracted chitin was thereafter converted to chitosan by deacetylation with $40 \% \mathrm{NaOH}$ for $2 \mathrm{~d}$. The resulting chitosan was ground into a fine powder (particle size $<1 \mathrm{~mm}$ ) by extensive grinding in an ultra centrifuge mill (Retsch, Germany).

Antifungal activity of irradiated chitosan powder: Chitosan powder was irradiated by gamma rays from a ${ }^{60} \mathrm{Co}$ source (Atomic Energy Authority) at a dose rate of $5 \mathrm{kGy} / \mathrm{h}$. A triplicate of each pack ( $3 \mathrm{~g}$ ) was subjected to different irradiation doses such as 25, 50, 75, 100 and $150 \mathrm{kGy}$. Chitosan powder ( $3 \mathrm{~g}$ ) without irradiation was used as the control. The colour of the irradiated chitosan powder was determined using a Minolta Chromameter (CR 200, Japan). Lightness (L) values were measured and the degree of change for each colour index, such as delta ' $L$ ' was expressed as the difference between value of control and value after irradiation at a specific dose ${ }^{11}$.
Each sample (1 g) was dissolved in $10 \%(\mathrm{v} / \mathrm{v})$ acetic acid $(100 \mathrm{~mL})$ and the $\mathrm{pH}$ of the solution was adjusted to 5.6 with $50 \%$ sodium hydroxide. Chitosan solution of $(1 \% \mathrm{w} / \mathrm{v})$ was obtained by dissolving $1 \mathrm{~g}$ of chitosan in $100 \mathrm{~mL} \mathrm{10 \%} \mathrm{(v/v)} \mathrm{glacial} \mathrm{acetic} \mathrm{acid} \mathrm{and} \mathrm{homogenizing}$ at $6500 \mathrm{rpm}$ for $5 \mathrm{~min}$ using IKA-Ultra-Turrax-T25 homogenizer. The $\mathrm{pH}$ of the solution was adjusted to 5.6 with $50 \% \mathrm{NaOH}$. The relative viscosity of each sample was measured using a rotational type viscometer (Fungi Lab Spain) at $25^{\circ} \mathrm{C}$ with the number 2 spindle at $60 \mathrm{rpm}$. The viscosity measurement was expressed in Centipoises (CP). The viscosity decrease rate (VDR) was calculated as $\operatorname{VDR}=\left(V / V_{0}\right) \times 100$, where $V_{0}$ was the viscosity of initial chitosan (non irradiated control) in $10 \%$ acetic acid solution and $V$ was the viscosity of the chitosan solution prepared from irradiated chitosan.

Two different strains of Colletortrichum gloeosporioides were isolated from diseased fruit of papaya var. Rathna and Red Lady respectively. Pathogenicity was proved in each case by establishing Koch's postulates. Pure cultures of respective strains were maintained separately on PDA at $25^{\circ} \mathrm{C}$. A mycelial disc $(0.9 \mathrm{~cm}$, diameter $)$ was cut from the peripheral region of $5 \mathrm{~d}$ old cultures of both fungal strains grown on PDA and transferred to the centre of $9 \mathrm{~cm}$ diameter, PDA plates. Aqueous solutions of chitosan prepared from irradiated powder (subjected to different doses) were incorporated separately into the medium at $50^{\circ} \mathrm{C}$ before plating. Plates incorporated with non-irradiated chitosan $(0 \mathrm{kGy})$ in $10 \%$ acetic acid and sterile distilled water (without chitosan) served as controls. Growth was assessed after $10 \mathrm{~d}$ by measuring the colony diameter in centimetres.

Antifungal activity of irradiated chitosan solution: Chitosan powder ( $1 \mathrm{~g})$ was dissolved in $10 \%$ acetic acid solution $(100 \mathrm{~mL})$, homogenized at $6000 \mathrm{rpm}$ for $5 \mathrm{~min}$ and filtered through muslin cloth. The resulting solution (Triplicate per irradiation dose, $100 \mathrm{~mL}$ per replicate) was irradiated directly by ${ }^{60} \mathrm{Co}$ gamma rays (Atomic Energy Authority) with doses of 5, 10, 25, 50, 75,100 and $150 \mathrm{kGy}$ at a dose rate of $5 \mathrm{kGy} / \mathrm{h}$. The $\mathrm{pH}$ of each solution was adjusted to 5.6 with $50 \%$ sodium hydroxide after the irradiation treatment. The colour and relative viscosity of the irradiated chitosan solutions were measured with a Chromameter (Minolta ,CR 200, Japan) and rotational type viscometer (FungiLab, Spain) at $25^{\circ} \mathrm{C}$ respectively. Antifungal activity of the irradiated chitosan solution on both fungal isolates was carried out as mentioned above. Plates incorporated with nonirradiated chitosan $(0 \mathrm{kGy})$ in $10 \%$ acetic acid and sterile distilled water (without chitosan) served as controls. 
Effect of irradiated chitosan on infection of fresh papaya fruits: Fresh papaya fruits from both Rathna and Red Lady varieties were harvested at colour index $2(10 \%$ yellow stage) from a commercial cultivation in Neelabamma, and transported to the laboratory on the same day. Twenty fruits of each variety were dipped in $4 \mathrm{~L}$ of $1 \%$ chitosan (non irradiated) for $5 \mathrm{~min}$ (T1) and another set was separately dipped in $1 \%$ chitosan solution irradiated at $5 \mathrm{kGy}$ (at a dose rate of $5 \mathrm{kGy}$ per h.) for $5 \mathrm{~min}$ (T2). After dipping, the fruits were allowed to air-dry ${ }^{12}$ for $5 \mathrm{~min}$ at $28^{\circ} \mathrm{C}$. Twenty fruits of each variety dipped in sterile distilled water for 5 min served as the control (T3). The treated and untreated fruits were then lined with styrofoam netting and placed vertically (stem end facing down) in corrugated fiberboard cartons. Each carton contained 10 fruits. The packaged fruits were stored at $13.5^{\circ} \mathrm{C}$ and $95 \% \mathrm{RH}$ for 2 and 3 wks. The fruits were removed from cold storage at the completion of the specified storage period, and ripened at $28^{\circ} \mathrm{C}$ using Ethral (ripening agent) in a ripening chamber. Ethral (1.6 $\mathrm{mL}$ ) was used in $93 \mathrm{~L}$ volume glass cubicles allowing $20 \%$ headspace. After ripening, fruits were held at $25^{\circ} \mathrm{C}$ for $24 \mathrm{~h}$. Overall visual quality was assessed according to the score based on disease severity (measuring the lesion diameter) and colour where $1-2$ = fruit not marketable, $3=$ poor quality but limited marketability, $4-5=$ fair quality and marketable, $6-7=$ good quality and marketable, $8-9=$ excellent quality $^{13}$. The percentage marketability was calculated as a ratio of the number of fruit with overall quality scores of $6-7$ or 8 - 9 against the total number of fruit used per treatment ${ }^{13}$.

Statistical method: The in vitro studies were conducted as a CRD (Completely Randomised Design) with ten replicates and repeated twice to confirm the results. Data were analyzed by analysis of variance and mean separation was done by Duncan's Multiple Range Test (DMRT) at $\mathrm{p}<0.05$. In vivo treatments were arranged in a completely randomized design (CRD) in the low temperature storage with 20 replicates per treatment. This experiment was repeated twice. Percentage marketability data were analyzed by a non-parametric Friedman two-way analysis (Minitab Inc., 1996).

\section{RESULTS}

\section{Antifungal activity of irradiated chitosan powder}

The change in colour of chitosan powder was calculated and presented as a 'Delta L' value. 'Delta L' $(\Delta L)$ value is widely adopted as a tool for measurement of browning of food $^{11}$. The colour of the powder changed to a more intense brown with increasing irradiation dose (Figure 1). It was observed that the browning discolouration at irradiation dose over $100 \mathrm{kGy}$ was not desirable. The relationship between the viscosity of the chitosan solution prepared from irradiated powder and irradiation dose is illustrated in Figure 2. Viscosity measurements are widely used to calculate the average molecular weight of chitosan ${ }^{9,11}$. Viscosity decrease rate was high between 0 to $75 \mathrm{kGy}$ but stabilized thereafter.

Significant growth $(\mathrm{p}<0.05)$ of the fungus (isolated from Red Lady) was observed in plates incorporated with chitosan irradiated at all doses including controls (Figure 3). However, the fungal strain isolated from Rathna behaved in a different manner and showed no mycelial growth in plates incorporated with $0 \mathrm{kGy}$ and 25 kGy chitosan solutions (prepared from irradiated powder) while control plates (without chitosan) were covered with mycelial growth at the end of 5day incubation (Figure 3). A significant $(p<0.05)$ increase in growth of the fungus (isolated from Rathna) was observed in plates incorporated with chitosan irradiated at 50, 75, 100 and 150 kGy doses (Figure 3).

\section{Antifungal activity of irradiated chitosan solution}

No significant $(\mathrm{p}<0.05)$ browning was observed in chitosan solutions irradiated at dose rates 5 and $10 \mathrm{kGy}$ compared to the original sample (control) as shown in Figure 4. However, browning increased thereafter with the increase of irradiation dose. The $100 \mathrm{kGy}$ treated sample showed the highest browning (high $\Delta \mathrm{L}$ value). Viscosity decrease rate (VDR \%) was higher from 0 to 5 $\mathrm{kGy}$ and stabilized at 5, 10 and $25 \mathrm{kGy}$ doses (Figure 5). A slight decrease in VDR was observed again from 25 to $50 \mathrm{kGy}$ and stabilized thereafter.

No fungal growth was observed with either of the isolates in plates incorporated with chitosan solutions irradiated at 5, 10, 25, 50, 75 and $100 \mathrm{kGy}$ and incubated at $28^{\circ} \mathrm{C}$ for 10 days (Figure 6). Maximum growth of the pathogen was observed in control plates. However, the plates incorporated with non-irradiated chitosan (0 kGy) and inoculated with the fungus isolated from Red Lady papaya showed a slight mycelial growth compared to the Rathna isolate. As the $1 \%$ chitosan irradiated in aqueous solution was found to be highly effective in controlling both isolates of $C$. gloeosporioides in vitro, it was decided to select this concentration for further in vivo experiments with fresh papaya. No significant $(p<0.05)$ growth of the fungus was observed when the irradiation dose was increased from 5 to $100 \mathrm{kGy}$. Therefore, $5 \mathrm{kGy}$ was selected as the effective dose for in vivo application for fresh papaya. 


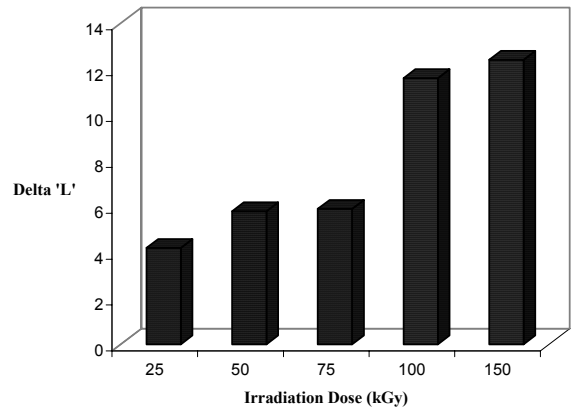

Figure 1: Change in Delta ' $L$ ' values (colour in relation to non irradiated controls-' L' value $=70$ ) of the irradiated chitosan powder

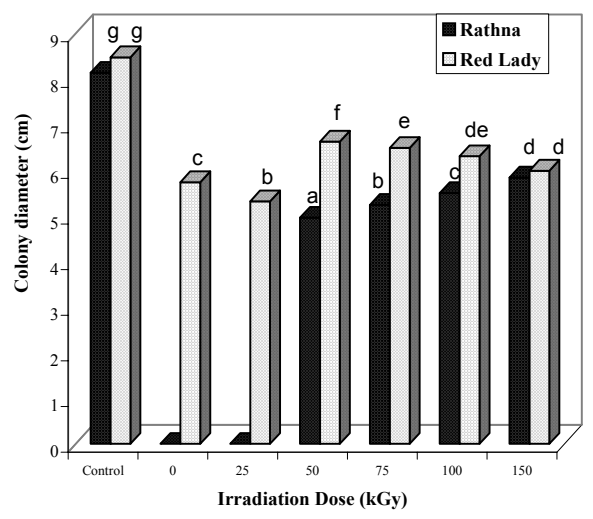

Figure 3: Antifungal activity of irradiated chitosan powder on the growth of anthracnose causing fungi isolated from Rathna and Red Lady papaya incubated for 5 days at $28^{\circ} \mathrm{C}$ (control $=$ in distilled water, $0=$ in non irradiated chitosan)

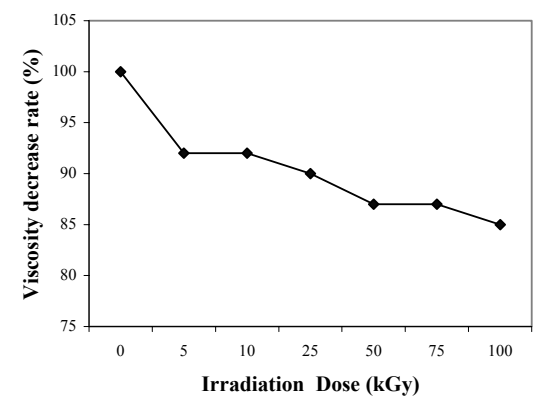

Figure 5: Effect of irradiation on viscosity decrease rate of chitosan solution directly irradiated at doses ranging from $0-100 \mathrm{kGy}$

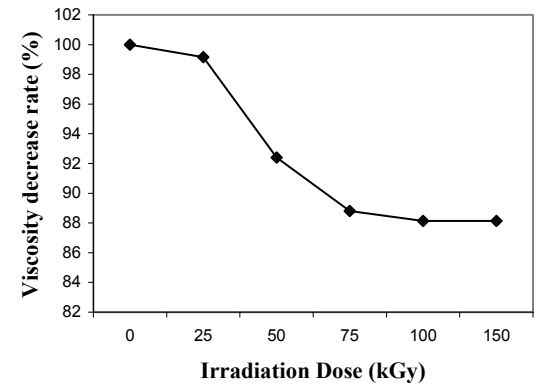

Figure 2: Effect of irradiation on viscosity decrease rate of chitosan powder in $10 \%$ aceticacid solution - dose range from 0 - $150 \mathrm{kGy}$

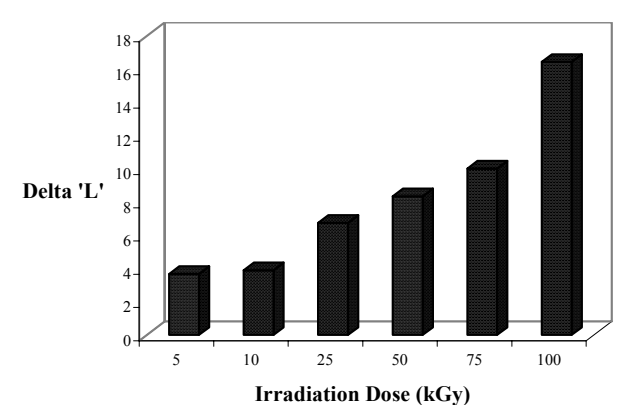

Figure 4: Change in Delta ' $L$ ' values (colour in relation to non irradiated controls-' $\mathrm{L}$ ' value $=50$ ) of the irradiated chitosan solution

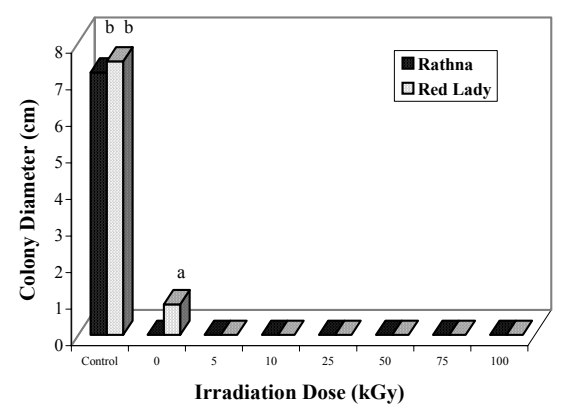

Figure 6: Antifungal activity of irradiated chitosan solution on the growth of anthracnose causing fungi isolated from Rathna and Red Lady papaya and incubated for 5 days at $28{ }^{\circ} \mathrm{C}$ (control $=$ in distilled water, $0=$ in non irradiated chitosan) 


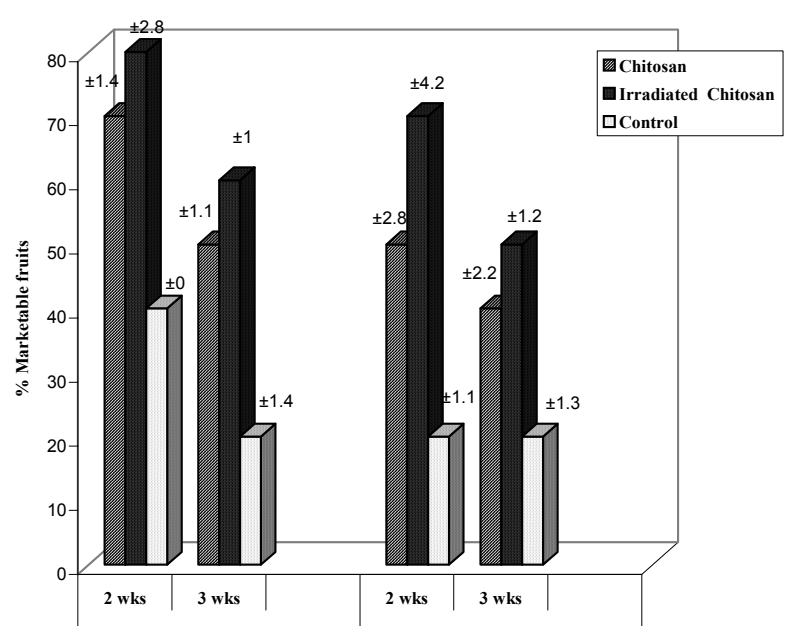

Figure 7: Percentage of the marketable fruit of papaya var. Rathna and Red Lady in relation to storage period and treatments applied (with \pm Standard deviation)

\section{Effect of irradiated chitosan on infection of fresh papaya fruit}

Eighty percent marketable quality fruit was observed in Rathna papaya subjected to irradiated chitosan dip treatment while non-irradiated chitosan treated fruit showed $70 \%$ marketability after 2 weeks storage at $13.5^{\circ} \mathrm{C}$ (Figure 7). When storage period was extended by a further week the quantity of marketable fruit in both treatments was reduced by $20 \%$. Overall fruit quality was better with less disease severity (indicated as higher marketability) when fruits were treated with irradiated chitosan than non-irradiated chitosan. In var. Red Lady the percentage of marketable fruits was lower than in var. Rathna with respect to both irradiated (70\%) and nonirradiated $(50 \%)$ chitosan treatments. However, control fruit dipped in distilled water of both varieties showed least number of marketable quality fruit $(20 \%)$.

\section{DISCUSSION}

Low molecular weight chitosan has been demonstrated to be an effective plant protector against infectious diseases and environmental stress ${ }^{11}$. It is also reported that the molecular weight and molecular fraction of chitosan, influence the solubility and interaction with the cell walls of target micro-organisms ${ }^{5}$. Ionization radiation is known as a useful tool for degradation of chitosan polymer ${ }^{14}$ and reducing molecular weight ${ }^{15}$. Present results indicated that the \% VDR of chitosan solutions decreased rapidly when subjected to lower irradiation doses and it decreased or stabilized with the increase in irradiation dose. Similar results were also obtained from irradiation treatment of chitosan and chitosan oligomer in $2002^{11}$. The rapid decrease in VDR with a low dose of irradiation (5 - 25 kGy) observed in the present study could be due to the irradiation-induced scissions of 1 - 4 glycosidic bonds of chitosan polysaccharide that caused a reduction of the molecular weight of the polymer ${ }^{11}$. An irradiation dose of over $75 \mathrm{kGy}$ was not suitable due to the development of an intense brown colour in chitosan solution that can affect the cosmetic appearance of coated fruits.

Chitosan solution (1\%) prepared from irradiated chitosan powder was found to be less effective in controlling the growth of $C$. gloeosporioides irrespective of the irradiation dose applied. Significant $(p<0.05)$ increase in the growth of the fungus was observed when irradiation dose was increased from $50 \mathrm{kGy}$ to $150 \mathrm{kGy}$ in irradiated chitosan solutions prepared from irradiated powder. Similar observations were made with increasing irradiation dose over $500 \mathrm{kGy}$ with respect to antimicrobial activity of irradiated chitosan powder by other scientists ${ }^{16}$. However, chitosan solution $(1 \%)$ directly subjected to irradiation doses even at $5 \mathrm{kGy}$ was found to be highly effective in in vitro control of both anthracnose causing fungal strains isolated from Rathna and Red Lady papaya. Degradation of chitosan by irradiation in aqueous solution is reported as an indirect effect due to the generation of hydroxyl radicals which in turn attack chitosan molecules by $\mathrm{H}$-abstraction, thus forming radicals at carbon atoms ${ }^{15}$. It is also reported ${ }^{15}$ that irradiation in solution (at $10 \mathrm{kGy}$ ) allows for easy and fast reduction of the molecular weight down to oligomer range. Higher antifungal activity seen in the present study with respect to the directly irradiated chitosan solution could be due to the above reasons. However, irradiation treatment with very high doses could break most of the bonds leading to complete degradation with monomer and short-chain oligomers. These fragments may not create inhibition effects on the growth of micro-organisms and they might even stimulate the growth as observed in some plate-culture conditions. These findings are consistent with the work reported in previous studies ${ }^{16}$. The differential response shown by the two fungal strains (isolated from Rathna and Red Lady) to irradiated chitosan could be due to the variation in cell wall characteristic (thickness) of these two fungal strains.

Previous studies conducted on the biological activity of chitosan suggested two different mechanisms of chitosan and target organism interactions. The first mechanism describes adsorption of chitosan to cell walls leading to cell walls covering, membrane disruption and cell leakage. The second mechanism proposed is the penetration of chitosan into living cells leading to inhibition of various enzymes and interference with 
synthesis of mRNA and proteins ${ }^{17-19}$. The action of original (non-irradiated) chitosan is likely to be the first mechanism that is the adhesion of the macromolecules to the cell wall of the target organism ${ }^{16}$. However, ionization radiation breaks the glycosidic links of chitosan to produce lower molecular weight fragments of varying size. The microbial inhibition caused by radiation-formed chitosan fragments could be stronger than that by original (non irradiated) molecules due to the contribution of both mechanisms (adsorption to cell wall and penetration to the cell) that occurred simultaneously in irradiated chitosan ${ }^{16}$. Irradiation of chitosan in the solid state leads to chain scission, and also causes side reactions such as oxidation and deamination ${ }^{15}$.

In the in vivo study, $80 \%$ fruit with marketable quality was observed when Rathna papaya was subjected to irradiated chitosan treatment while non-irradiated chitosan treated fruit showed $70 \%$ marketability after 2 weeks storage at $13.5^{\circ} \mathrm{C}$. Sixty percent fruit with marketable quality was observed even when storage life was extended for 3 weeks with irradiated chitosan treated Rathna papaya. It was observed that irradiated chitosan treatment (70\% marketable fruit) was suitable for Red Lady variety where non-irradiated chitosan treatment (50\% marketable fruit) was less effective in controlling the anthracnose disease of this variety. In vivo studies with fresh Rathna and Red Lady papayas showed that shelf life could be extended up to 3 weeks (with $60 \%$ marketable fruit) when chitosan solution is subjected to irradiation treatment at $5 \mathrm{kGy}$.

\section{Acknowledgement}

The authors gratefully acknowledge the financial assistance provided by the Industrial Technology Institute, Treasury Grant (10717 / TG8) and Norwegian Agency for Development Corporation (NORAD). We thank the Atomic Energy Authority (AEA) for allowing us to use the irradiation facility.

\section{References}

1. El-Ghaouth A., Arul J., Wilson C. \& Benhamou N. (1997). Biochemical and cytochemical aspects of the interaction of chitosan and Botrytis cenera in bell pepper fruit. Postharvest Biology and Technology 12:183-194.

2. Hirano S. et al. (1990). Chitosan as an ingredient for domestic animal feeds. Journal of Agriculture and Food Chemistry 38 : 1214 - 1217.

3. Hewajulige I.G.N., Sivakumar D., Sultanbawa Y., Wilson Wijeratnam R.S. \& Wijesundara R.L.C. (2006). Effect of chitosan coating on postharvest life of papaya (Carica papaya L.) var. Rathna grown in Sri Lanka. Tropical Agricultural Research 18: 135 - 142
4. Hewajulige I.G.N., Sivakumar D., Sultanbawa Y., Wilson Wijeratnam R.S. \& Wijesundara R.L.C. (2007). Effect of chitosan coating on the control of anthracnose and overall quality retention of papaya (Carica papaya L.) during storage. Acta Horticulturae 740:245 - 250.

5. Tikhonov V.E. et al. (2005). Bactericidal and antifungal activities of low molecular weight chitosan and its N-/2 (3) - (dodec-z-enyl) succinoyl/ - derivatives. Carbohydrate polymers 64(1):66-72.

6. Clelend M.R., Park L.A. \& Cheng S. (2003). Application for radiation processing of materials. Nuclear Instrument Methods B 208:66 - 73 .

7. Ulanski P. \& Rosiak J.M. (1992). Preliminary studies on radiation-induced changes in chitosan. Radiantion Physics and Chemistry 39(1):53 - 57.

8. Ramnani S.P., Chaudhari N.D., Patil S. \& Sabharwal S. (2004). Synthesis and characterization of crosslinked chitosan formed by $\gamma$ irradiation in the presence of carbontetrachloride as a sensitizer. Journal of Polymer Science Part A Polymer Chemistry 42: 3897 - 3909.

9. Lim L.Y., Khor E. \& Koo O. (1998). Gamma irradiation of chitosan. Journal of Biomedical Materials Research 43:282 - 290.

10. Chvajarempum J. (2002). Gamma radiation degradation of chitosan for use as plant growth promoter and fungicides, Abs. Country paper (Thailand), FNCA workshop on application of electron acceleration, Japan.

11. Won-Seok C., Kil-Jin A., Dong-Wook L., Myung-Woo B. \& Hyun-Jin P. (2002). Preparation of chitosan oligomers by irradiation. Polymer Degradation and Stability 78:533-538.

12. Fallik E., Grinberg S. \& Ziv O. (1997). Potassium bicarbonate reduces postharvest decay development bell pepper fruits. Journal of Horticultural Science 72:35-41.

13. Sivakumar D., Hewarathnagamagae N.K., Wilson Wijeratnam R.S. \& Wijesundara R.L.C. (2002). Effect of ammonium carbonate and sodium bicarbonate on anthracnose of papaya. Phytoparasitica 30(5):486 - 492

14. Woods J.R. \& Pikaev A.K.(Eds.). (1994). Applied Radiation Chemistry: Radiation Processing, Wiley International Publishers, New York.

15. Czechowska-Biskup R., Rokita B., Ulanski P. \& Rosiak J. M. (2005). Radiation-induced and sonochemical degredation of chitosan as a way to increase its fat-binding capacity. Nuclear Instruments and Methods in Physics Research B 236:383 - 390.

16. Lam N.D. \& Diep T.B. (2003). A preliminary study on radiation treatment of chitosan for enhancement of antifungal activity tested on fruit-spoiling strains. Nuclear Science and Technology 2(2):54-60.

17. Rebea E.I., Badawy M.E.T., Stevens C.V., Samagghe G. \& Steurbaut W. (2003). Chitosan as antimicrobial agent: applications and mode of action. Biomacromolecules 4(6): 1457-1465.

18. Chirkov S.N. (2002). The antiviral activity of chitosan. Applied Biochemistry and Microbiology 38(4):1-8

19. Zheng L.Y. \& Zhu J.F. (2003). Study on antimicrobial activity of chitosan with different molecular weights. Carbohydrate Polymers 54:527-530. 